\title{
Vascular Changes in the Various Substrains of Spontaneously Hypertensive Rats, Untreated and Treated with Sodium Chloride in Diet
}

\author{
Fumitada Hazama, M.D., Akira Ooshima, M.D., Kazuhiko \\ Tomimoto, M.D., and Kozo Oкамото, M.D.
}

In preliminary experiments it was found that the spontaneously hypertensive rat (SHR) given $1 \%$ saline for drinking in place of tap water developed a high incidence of cerebrovascular diseases, extensive vascular changes in the kidneys, heart etc, and that there were definite differences in the incidence of these pathological changes among the substrains of SHR (Okamoto et al : In "Spontaneous Hypertension ", Igaku Shoin, Tokyo, p 129, 1972. Hazama et al: ibid, p 134). The purposes of the present study are to determine the dietary effects of sodium chloride on hypertensive vascular changes in detail and to identify the differences among pathological changes of various substrains.

SHR, which belong to the substrains $A_{1}, A_{2}, A_{3}, B$, and $C$, were alloted into 2 groups. One group was fed on normal stock chow with ordinary drinking water (control group). To the second group, $1 \%$ saline was given for drinking ad libitum after 1.5-2 months of age ( $\mathrm{NaCl}$ group). Histological study was carried out on various organs after a natural death in these animals. The brains were cut into 4 or 5 frontal sections, and the localization of changes was carefully studied.

Animals in the $\mathrm{NaCl}$ group began to show neurological signs approximately 5 weeks after initiation of salt loading such as involuntary movements, disturbance in gait, paresis or paralysis of the extremities, incontinentia urinae, rage etc. The brains of these animals often showed macroscopical findings, massive intracerebral hemorrhage or an infarct. In the hemorrhagic focus a thin-walled small artery was often observed, which proved to be angiographically microaneurysm. Old foci of infarcts took the form of cyst or scar. In and near these foci small arteries and arterioles showed various changes such as angionecrosis, hyaline degeneration, narrowing of the lumen due to thickening of the wall, thrombotic obstruction etc.

Both hemorrhage and infarct occurred at similar sites in the brain, that is parasagittal cortex and subcortex. These sites of cerebrovascular diseases 
correspond to the borders between the medial and anterior or posterior cerebral arteries. In the control group the vascular diseases occurred mostly later than 200 days of age. On the other hand, the animals in the $\mathrm{NaCl}$ group showed cerebrovascular changes between 35 and 140 days after the initiation of $\mathrm{NaCl}$ treatment, that is between 90 and 200 days of age. The animals in the substrain $\mathrm{G}$ were an exception. Vascular changes before 200 days of age were not seen.

In the control group the incidences of cerebrovascular diseases in the substrains $\mathrm{A}_{1}, \mathrm{~A}_{2}, \mathrm{~A}_{3}, \mathrm{~B}$, and $\mathrm{C}$ were $27.0,8.3,45.9,4.3$, and $4.0 \%$ respectively. The incidence in $\mathrm{A}_{3}$ is significantly high when compared with other substrains. On the other hand the incidences of cerebrovascular diseases in the $\mathrm{NaCl}$ group were increased. The incidences in $A_{1}, A_{2}, A_{3}, B$, and $C$ were 76.0, 58.8, 82.4, 64.9, and $11.8 \%$ respectively. The differences in incidence between $\mathrm{C}$ and each of the remaining substrains were statistically significant. This suggests that the substrain $C$ is less sensitive to sodium chloride, while the remainings are highly sensitive to $\mathrm{NaCl}$.

Some SHR showed severe pathological changes in the kidneys, though the incidence was low. The basement membranes of the glomerular capillaries were in general thickened and partially necrotic. Arterioles and small arteries revealed fibrinoid necrosis and cellular hyperplasia. These findings correspond to those of malignant hypertension. The incidences of fibrinoid necrosis in $A_{1}, A_{2}, A_{3}, B$, and $C$ were $23.0,8.2,15.0,1.4$, and $7.7 \%$ respectively. In the $\mathrm{NaCl}$ group, pathological renal changes occurred very early and the incidence was very high. The incidences of fibrinoid necrosis in $A_{1}, A_{2}, A_{3} B$, and $\mathrm{C}$ were $77.3,52.9,87.5,54.1$, and $25.0 \%$ respectively. The differences in incidence between $A_{3}$ and $A_{2}, B$ or $C$ were statistically significant. Here the substrain $\mathrm{C}$ was revealed to be less sensitive to sodium chloride.

In the heart diffuse fibrosis, scar formation, fibrinoid necrosis of arterioles and small arteries often associated with cellular hyperplasia were found. The differences in incidence of these changes among the substrains were not so marked as in the kidney or brain. These findings were not exaggerated by salt loading except for fibrinoid necrosis.

\section{Summary}

(1) There were definite differences in incidence of the hypertensive vascular changes in the brain and kidneys among the substrains of SHR.

(2) The loading of sodium chloride in drinking water accelerates the development of hypertensive vascular changes and sequential pathological changes in the brain and kidneys of SHR.

(3) The substrains $A_{3}$ and $C$ of SHR have a peculiar nature with regard 
to cerebrovascular diseases. $A_{3}$ develops spontaneously cerebrovascular diseases with a high incidence, and shows the highest incidence of the changes among various substrains by salt loading. The substrain $\mathrm{C}$ shows the lowest incidence of cerebrovascular diseases in the nontreated animals as well as in the $\mathrm{NaCl}$ group. This substrain does not appear to be salt-sensitive.

(4) These facts suggest that inherited factors play an important role in developing hypertensive vascular diseases. 